\title{
miR-132 in atrial fibrillation directly targets connective tissue growth factor
}

\author{
GANG QIAO, DONGSHENG XIA, ZHAOYUN CHENG and GUOBAO ZHANG \\ Department of Cardiovascular Surgery, Henan Provincial Hospital, Zhengzhou University, \\ Zhengzhou, Henan 450003, P.R. China
}

Received August 24, 2016; Accepted May 16, 2017

DOI: $10.3892 / \mathrm{mmr} .2017 .7045$

\begin{abstract}
Atrial fibrillation (AF) is the most frequently occurring, persistent cardiac arrhythmia, and the hallmark of AF-dependent structural remodeling is atrial fibrosis. Connective tissue growth factor (CTGF) is important in the process of fibrosis. The association between miRNA and CTGF in AF-dependent fibrosis remains to be elucidated. The present study aimed to determine if microRNA (miR)-132 was able to regulate CTGF with an anti-fibrotic effect in AF. A total of ten dogs or patients were assigned to control $(n=4)$ and AF groups $(n=6)$. The left atrium of dogs or right atrial appendage of patients was observed. Following this, cardiac fibroblasts of adult rats were treated with or without angiotensin II (Ang II). Furthermore, cardiac fibroblasts were transfected with miR-132 mimics, inhibitor or negative control. The expression of miR-132 and CTGF were analyzed by reverse transcription-quantitative polymerase chain reaction or western blotting. These analyses demonstrated that miR-132 expression was decreased and CTGF increased in the human and canine models with AF. The expression of miR-132 and CTGF protein levels were upregulated in Ang II stimulated cardiac fibroblasts of adult rats. Furthermore, when miR-132 was introduced into cardiac fibroblasts, the expression of miR-132 increased significantly whereas the expression of CTGF decreased. Inverse results were observed when cardiac fibroblasts were transfected with miR-132 inhibitor. The luciferase reporter assay was then performed to confirm that miR-132 may suppress CTGF expression by binding to its 3'-untranslated region. In conclusion, miR-132 may target CTGF in regulating fibrosis in Ang II-treated cardiac fibroblasts. These findings may aid in providing potential therapeutic targets in the treatment of AF.
\end{abstract}

Correspondence to: Dr Guobao Zhang, Department of Cardiovascular Surgery, Henan Provincial Hospital, Zhengzhou University, 7 WeiWu Road, Zhengzhou, Henan 450003, P.R. China E-mail: zhang_gb68@163.com

Key words: atrial fibrillation, fibrosis, microRNAs, connective tissue growth factor, angiotensin II

\section{Introduction}

Atrial fibrillation (AF) is the most frequently occurring and persistent cardiac arrhythmia (1). Electrophysiological and structural remodeling contributes to the generation of AF (2). Atrial fibrosis is a hallmark of structural remodeling in the heart (3) and AF is promoted by atrial fibrosis (4). Cardiac fibroblasts (CFs) account for $70 \%$ cells in the heart (5). CFs synthesize extracellular matrix (ECM) proteins to develop cardiac fibrosis (6). Furthermore, it has been demonstrated that the rennin-angiotensin system is important in the occurrence of AF (7). Angiotensin II (Ang II) appears to be associated with $\mathrm{AF}$, which has a key role in cardiac remodeling and fibrosis through the promotion of cell proliferation and ECM synthesis $(8,9)$. Increasing evidence suggests numerous regulated factors may participate in atrial fibrosis (10).

MicroRNAs (miRNAs) are small non-coding RNAs of 18-25 nucleotides, which bind to the 3'untranslated region (UTR) of target mRNAs to block translation or induce degradation of mRNA (11). Accumulating evidence demonstrates that miRNAs are important in the pathogenesis of fibrosis (12). miR-21 has previously been demonstrated to be associated with fibrosis of the atrial myocardium by targeting Sprouty 1 (13). miR-133 and miR-30 are anti-fibrotic and control the structural alterations that are evident in chronic $\mathrm{AF}$ (14). miR-29 has been hypothesized to enhance the cardiac fibrotic response (15). miR-132 is transcribed under the regulation of cAMP response element binding protein (CREB), which is a known Ang II regulated gene (16). Ang II upregulates the levels of miR-132 in CFs (17). A previous study demonstrated that miR-132 is involved in the vascular smooth muscle dysfunction mediated by Ang II (18). The overexpression of miR-132 in pancreatic adenocarcinoma tissues stimulates the proliferation of pancreatic cancer Panc-1 cells (19). The inhibition of miR-132 in human lung cancer H1299 cells may reduce tumor tissue proliferation and angiogenesis (20). However, the role of miR-132 in fibrosis remains largely unknown.

Connective tissue growth factor (CTGF) has been demonstrated as a secreted protein and may promote ECM synthesis (21). The induction of ECM synthesis occurs in parallel with the generation of fibrosis (22). It has previously been identified that CTGF is a potent pro-fibrotic factor in AF (23). CTGF may be upregulated and may subsequently induce atrial fibrosis in AF patients via the tumor growth factor 
(TGF)- $\beta 1$ and mothers against decapentaplegic signaling pathways (24). In addition, Ang II induces cardiac fibrosis and is widely regarded as an important amplifier of the profibrogenic actions of TGF- $\beta 1$ in a variety of tissues (25). Furthermore, CTGF is a downstream factor of TGF- $\beta 1$ in the setting of Ang II-induced cardiac fibrosis (26). CTGF may be induced by Ang II in the hearts of perfused rats (23). A previous study revealed that CTGF is regulated by two primary cardiac miRNAs, miR-133 and miR-30 (27). However, regulation of CTGF by miRNA functioning in fibrosis has not yet been identified.

The present study hypothesized that miR-132 may regulate the CTGF gene involved in fibrosis. Reverse transcription-quantitative polymerase chain reaction (RT-qPCR) and western blot analysis revealed that miR-132 targeted CTGF and corresponding mRNA and protein expression levels were detected in canine and human pathological models with AF. The role of miR-132 in inhibiting fibrosis by targeting CTGF was verified. The findings of the present study provide a novel mechanism in AF, where the upregulation of the expression of CTGF and downregulation of miR-132, may suggest a molecular mechanism associated with the development of AF-dependent fibrosis.

\section{Materials and methods}

Animal studies. The animal experiments in the present study were approved by the Animal Care and Use Committee of Henan Provincial Hospital, Zhengzhou University (Zhengzhou, China). All protocols were conducted following guidelines of the National Animal Research Center.

Beagle dogs ( $n=10,12-16$ months of age, female and male, body weight $10.5 \pm 2.3 \mathrm{~kg}$ ) were supplied by the Lab Animal Center of Henan Provincial Hospital, Zhengzhou University. Dogs were kept in separate cages with an ambient temperature of $24^{\circ} \mathrm{C}$ and $45 \%$ humidity, with a $14 \mathrm{~h}$ light/dark cycle and were fed a standard diet with ad libitum access to food and water.

A total of six dogs had an atrial pacemaker implanted, as previously described (28). Dogs were anesthetized using pentobarbital $(30 \mathrm{mg} / \mathrm{kg})$ and butorphanol $(0.3 \mathrm{mg} / \mathrm{kg})$. During the surgery, body temperatures were maintained at $>37^{\circ} \mathrm{C}$. Heart rate and rhythm were monitored using an electrocardiogram. A catheter was inserted in the right femoral artery to monitor the arterial blood pressure and arterial oxygen pressure. A total of two screw-in bipolar electrode leads were inserted via the right internal jugular vein and fixed to the right atrial (RA) appendage. The first electrode lead was tunneled subcutaneously to the posterior cervical region and exteriorized and the other was connected to an atrial pacemaker (600 bpm; SIP501; Star Medical Co., Ltd., Tokyo, Japan) in a subcutaneous pocket created in the neck. Atrioventricular conduction was preserved. The no-paced sham dogs $(n=4)$ received the same surgery without being implanted with an atrial pacemaker.

A total of 8 weeks following implantation, animals were euthanized by intravenous administration of sodium pentobarbital $(300 \mathrm{mg} / \mathrm{kg})$. Following this, the left atrium samples were removed from all the dogs. The samples were then washed with $0.9 \%$ saline to remove blood, placed into liquid nitrogen immediately and stored at $-80^{\circ} \mathrm{C}$ until further use.
Table I. Patient characteristics.

\begin{tabular}{lcc}
\hline & SR $(\mathrm{n}=4)$ & $\mathrm{AF}(\mathrm{n}=6)$ \\
\hline Age (years) & $60.25 \pm 3.69$ & $59.47 \pm 5.34$ \\
Sex & $2 \mathrm{M} / 2 \mathrm{~F}$ & $3 \mathrm{M} / 3 \mathrm{~F}$ \\
LA diameter $(\mathrm{mm})$ & $46.30 \pm 1.24$ & $52.60 \pm 1.75^{\mathrm{a}}$ \\
RA diameter $(\mathrm{mm})$ & $41.50 \pm 1.60$ & $51.20 \pm 1.43^{\mathrm{a}}$ \\
EF $(\%)$ & $57.41 \pm 1.62$ & $57.37 \pm 1.70$
\end{tabular}

There were no significant statistical differences between SR group and $\mathrm{AF}$ group in age and $\mathrm{EF}$. ${ }^{\mathrm{a}} \mathrm{P}<0.01$ vs. $\mathrm{SR}$. $\mathrm{AF}$, atrial fibrillation; $\mathrm{SR}$, sinus rhythm; EF, ejection fraction; LA, left atrium; RA, right atrium; M, male; F, female.

Patient studies. The right atrial appendage samples of 10 patients (5 male and 5 female) who had undergone coronary bypass surgery at the start of the investigation at Henan Provincial Hospital, Zhengzhou University (Henan, China) were used in the present study. Patients were selected to participate in the present study from September 2011 to November 2013. All patients were under the age of 65 , six were diagnosed with AF (AF group) and 4 with no history of AF [sinus rhythm (SR) group] (Table I). Furthermore, those who had severe renal or liver dysfunction, infective endocarditis, hyperthyroidism, hypertension or diabetes were excluded from the study. Prior to the establishment of coronary bypass surgery in patients, $150 \mathrm{mg}$ right atrial appendage specimens with an area $\sim 1.0 \times 0.5 \times 1.0 \mathrm{~cm}$ were taken. Patients with sinus rhythm served as controls $(n=4)$. The samples were washed with $0.9 \%$ saline to remove blood, placed into liquid nitrogen immediately and stored at $-80^{\circ} \mathrm{C}$ until further use. The study was approved by the Ethics Committee of the Henan Provincial Hospital, Zhengzhou University and informed written consent was obtained from patients.

Isolation and culture of rat cardiac fibroblasts. Twenty Sprague-Dawley (SD) rats (aged 6-8 weeks) were purchased from the Laboratory Animal Center of Shanghai Institutes for Biological Science (Shanghai, China). The male rats weighing 250-300 g were used to obtain cardiac fibroblasts. All rats were housed separately in a room where the temperature remained between $20-26^{\circ} \mathrm{C}$, relative humidity was $40-70 \%$, at a $12 \mathrm{~h}$ light/dark cycle and were fed a standard diet with ad libitum access to food and water.

The rats were deeply anesthetized with isoflurane (5\%) and sacrificed by cervical dislocation. Following rapid excision of the hearts of rats, fibroblasts were isolated by enzymatic digestion with a collagenase/trypsin solution. Following $2 \mathrm{~h}$ of attachment to the uncoated culture plates, the attached cells were washed and cultured in plating medium. Following 2 days of culture, the cells were amplified in Dulbecco's modified Eagle's medium (DMEM; Invitrogen; Thermo Fisher Scientific, Inc., Waltham, MA, USA) supplemented with $20 \% \mathrm{FBS}$ and incubated at $37^{\circ} \mathrm{C}$, in a humid chamber in an environment containing $5 \% \mathrm{CO}_{2}$. When the cells reached $80 \%$ confluence, the medium was removed and a serum-free DMEM medium added, containing $1 \mu \mathrm{mol} / 1$ Ang II (Sigma-Aldrich; Merck KGaA, Darmstadt, Germany). 
Table II. Primer sequences for polymerase chain reaction.

\begin{tabular}{lll}
\hline Species & Gene & Primer sequences \\
\hline Mouse & miR-132 & F: GCTCTCATCGCTAGCGATCGTAG \\
Mouse & CTGF & R: TAACAGTCTACAGCCATGGTCG \\
Human & miR-132 & R: GGCAGTTGGCTCTAATCATAGTTGGG \\
Human & & F: CGGCGGTAACAGTCTACAGCCA \\
Dog & CTGF & R: GTGCAGGGTCCGAGGT \\
Dog & miR-132 & R: CCCTGCATCTTCGGTGGTA \\
& & F: GATACGCCCGTCTACTGTTTC \\
& CTGF & R: GGCTGTAGACTGTTACCAAAA \\
\hline
\end{tabular}

F, forward; R, reverse; miR-132, microRNA-132; CTGF, connective tissue growth factor.

In order to decide the optimal condition of Ang II for cell culture, cells were treated with different Ang II concentrations (including $10^{-8}, 10^{-7}, 10^{-6}, 10^{-5} \mathrm{~mol} / \mathrm{l}$ ) for $24 \mathrm{~h}$ and the expression of miR-132 and CTGF confirmed that $10^{-7} \mathrm{~mol} / \mathrm{l}$ was the optimal concentration. Cells were then treated for differing time lengths (including 6, 12, 24 and $48 \mathrm{~h}$ ) with $10^{-7} \mathrm{~mol} / \mathrm{l}$ Ang II the expression levels of miR-132 and CTGF were detected to verify that $24 \mathrm{~h}$ was the optimal time. Cells were treated with $10^{-7} \mathrm{~mol} / \mathrm{l}$ Ang II for $24 \mathrm{~h}$ and cells cultured in the medium without Ang II were used as control. Cells were then harvested and stored at $-80^{\circ} \mathrm{C}$ for further study.

Transfection. The mouse miR-132 mimics, inhibitors and scramble miR negative control (NC) were purchased from Invitrogen (Thermo Fisher Scientific, Inc.) and were introduced into cardiac fibroblasts of SD rats at a final concentration of $50 \mathrm{nM}$. All cell transfections were performed using Lipofectamine 2000 (Invitrogen; Thermo Fisher Scientific, Inc.) according to the manufacturer's protocol. Following $48 \mathrm{~h}$ of transfection, cells were harvested for further analysis. The sequences of miR-132 mimics, inhibitors and scramble miR NC are as follows: 5'-UAACAGUCUACAGCCAUGGUCGAC CAUGGCUGUAGACUGUUAUU-3' for miR-132 mimics; 5'-CGACCAUGGCUGUAGACUGUUA-3' for miR-132 inhibitors and 5'-CAGUACUUUUGUGUAGUACAA-3' for scramble miR NC.

$R T-q P C R$. Total RNA was extracted using TRIzol ${ }^{\circledR}$ (Invitrogen; Thermo Fisher Scientific, Inc.) according to the manufacturer's protocol (29). cDNA was then synthesized via reverse-transcription using a cDNA synthesis kit (Takara Bio, Inc., Otsu, Japan). The RT-qPCR was performed using a SYBR PrimeScript miRNA RT-PCR kit (Takara Bio, Inc.) on an ABI 7900 thermocycler (Applied Biosystems; Thermo Fisher Scientific, Inc.). Cycling conditions were as follows: An initial predenaturation step at $50^{\circ} \mathrm{C}$ for $2 \mathrm{~min}$ and $94^{\circ} \mathrm{C}$ for 10 min followed by 40 cycles of denaturation at $94^{\circ} \mathrm{C}$ for $15 \mathrm{sec}$, annealing at $58^{\circ} \mathrm{C}$ for $15 \mathrm{sec}$ and elongation at $72^{\circ} \mathrm{C}$ for $30 \mathrm{sec}$. U6 small RNA or GAPDH was used as an internal control. The relative expression of mRNA or miRNA was calculated by the $2^{-\Delta \Delta \mathrm{Cq}}$ method (30). The primers used in the current study are presented in Table II.

Western blotting. The methods used in the present study for western blot analysis were conducted as previously described (31). In brief, tissues or cells were lysed in NP-40 buffer containing $50 \mathrm{mM}$ Tris- $\mathrm{HCl}, \mathrm{pH} 7.4,150 \mathrm{mM} \mathrm{NaCl}$, $1 \%$ NP-40, 1 mM EDTA and protease inhibitor (Roche Diagnostics, Basel Switzerland). The protein concentration was quantified using a bicinchoninic acid assay kit (Beyotime Institute of Biotechnology, Haimen, China). Subsequently, the proteins $(40 \mu \mathrm{g})$ were separated by $10 \%$ SDS-PAGE and then transferred to a polyvinylidene membrane (EMD Millipore, Billerica, MA, USA). The membranes were blocked for $1 \mathrm{~h}$ at room temperature using 5\% non-fat milk. Following this, the membranes were incubated with primary antibodies against CTGF (1:500; cat. no. sc-365970; Santa Cruz Biotechnology Inc., Dallas, TX, USA) or GAPDH (1:1,000; cat. no. 3683; Cell Signaling Technology, Inc., Danvers, MA, USA) overnight at $4^{\circ} \mathrm{C}$. After that, the membranes were washed 3 times for $10 \mathrm{~min}$ each with Tris-buffered saline containing $0.1 \%$ Tween (TBS-T), followed by incubation with horseradish peroxidase-conjugated IgG secondary antibodies (1:5,000; cat. no. sc-280786; Santa Cruz Biotechnology, Inc.) for $1 \mathrm{~h}$ at room temperature. Blots were visualized by ECL Detection Reagents (GE Healthcare Life Sciences, Little Chalfont, UK) and the expression level of CTGF protein was calculated by densitometry of CTGF, relative to that of GAPDH. Densitometry of the bands was quantified with ImageJ software version 1.47 (National Institutes of Health, Bethesda, MA, USA).

Luciferase reporter assay. Total cDNA from cardiac fibroblasts was used to amplify the 3'UTR of CTGF by PCR. The CTGF 3'UTR was cloned into pGL3 luciferase reporter (Promega Corporation, Madison, WI, USA), yielding pGL3-CTGF. Mutations were introduced in potential miR-132 binding sites using the QuikChange site-directed mutagenesis kit (Agilent Technologies, Inc., Santa Clara, CA, USA). Following this, 

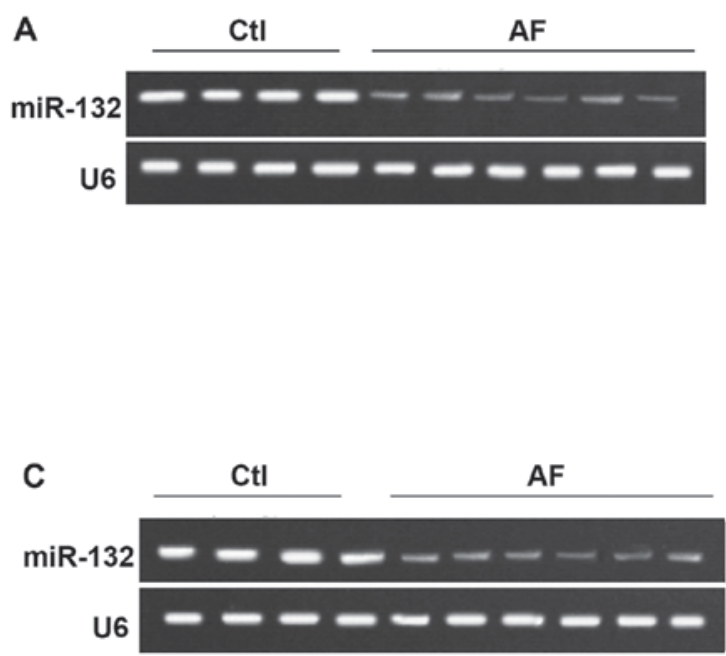

B
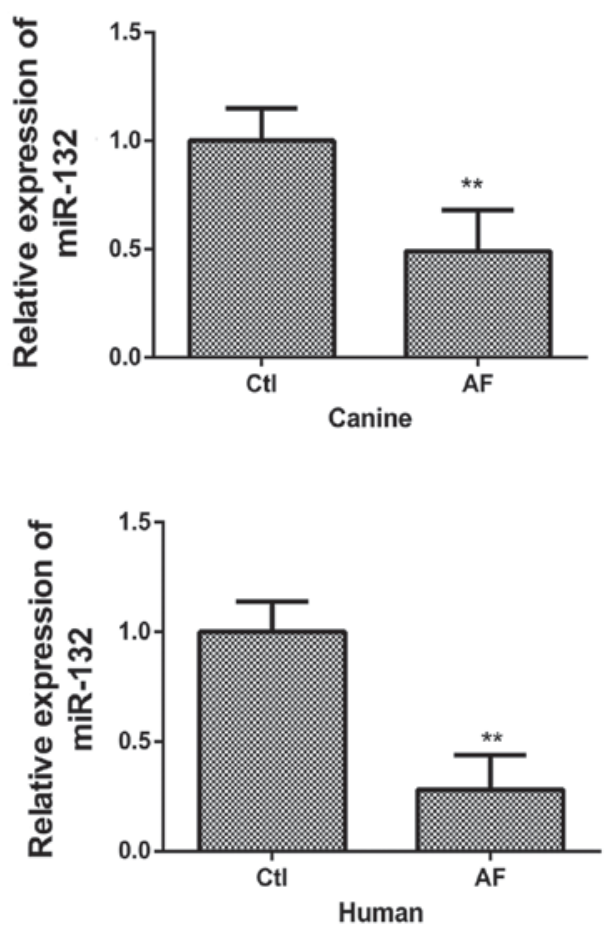

Figure 1. miR-132 is downregulated in the canine and human samples with AF, detected by reverse transcription-quantitative polymerase chain reaction. (A) Expression levels of miR-132 in the left atrium of dogs with or without AF. (B) A graphical representation of miR-132 expression profiles. (C) Expression levels of miR-132 in the right atrial appendage of patients with or without AF. (D) A graphical representation of miR-132 expression profiles. Dogs with AF induced by animal pacemaker $(n=6)$, no-paced sham dogs served as control $(n=4)$. Patients with $A F(n=6)$, patients with sinus rhythm served as control $(n=4)$. Representative gels were from 6 different AF samples and 4 control samples. Values were normalized to U6. Data are represented as the mean \pm standard deviation of the pooled samples. ${ }^{* *} \mathrm{P}<0.01$ vs. control group. AF, atrial fibrillation; miR-132, microRNA-132; Ctl, control.

$1 \mu \mathrm{g}$ constructs were co-transfected with $1 \mu \mathrm{g}$ miR-132 mimic or NC into the cardiac fibroblasts using Lipofectamine 2000 (Invitrogen; Thermo Fisher Scientific, Inc.) according to the manufacturer's protocol. At $48 \mathrm{~h}$ post-transfection, luciferase activities were determined using the Dual-Luciferase Reporter Assay system (Promega Corporation) according to the manufacturer's protocol. Reporter luciferase activity was normalized to the luciferase activity of the internal control Renilla.

Statistical analysis. Statistical data were presented as the mean \pm standard deviation. SPSS software, version 16.0 (SPSS, Inc., Chicago, IL, USA) was used for statistical analysis. Differences between two groups were evaluated by unpaired Student's t-test. Multigroup comparisons were conducted using two-way analysis of variance followed by Bonferroni post hoc tests. $\mathrm{P}<0.05$ was considered to indicate a statistically significant difference.

\section{Results}

miR-132 is downregulated in canine and human atrial fibrillation samples. In order to determine the expression of miR-132, RT-qPCR was performed in samples from dogs and patients with or without AF. As presented in Fig. 1A, the expression of miR-132 was significantly downregulated in the dogs with AF compared with control. Furthermore, statistical analysis verified that the relative expression of miR-132 decreased in the dogs with AF compared with control (Fig. 1B). It was additionally revealed that the expression of miR-132 in the patients with $\mathrm{AF}$ was decreased compared with control (Fig. 1C and D).
CTGF $m R N A$ and protein levels are upregulated in canine and human samples with atrial fibrillation. In order to detect the expression of CTGF, RT-qPCR and western blot analysis were conducted on samples from dogs and patients with or without AF. As presented in Fig. 2A, significant upregulation of CTGF mRNA expression was observed in the dogs with AF compared with control. Similar results were detected in the patients with AF (Fig. 2B). Furthermore, the protein expression levels of CTGF were increased significantly in the dogs with AF compared with control (Fig. 2C), a trend that was additionally observed in the patients with AF (Fig. 2D).

miR-132 and CTGF expression levels are upregulated in cardiac fibroblasts treated with Ang II. To evaluate the expression of miR-132 and CTGF in cardiac fibroblasts treated with Ang II, RT-qPCR and western blot analysis were carried out. As demonstrated in Fig. 3A, miR-132 increased in cardiac fibroblasts treated with Ang II compared with Ang II-non-treated negative control group. Furthermore, the statistical analysis revealed similar results (Fig. 3B). In addition, the protein expression levels of CTGF were upregulated significantly when cardiac fibroblasts were treated with Ang II, compared with the Ang II-non-treated negative control group (Fig. 3C and D).

miR-132 inhibits the expression of CTGF in cardiac fibroblasts. To detect the potential role of miR-132 in the expression of CTGF, the cardiac fibroblasts were transfected with miR-132 mimics, miR-132 inhibitor or NC vector. The introduction of miR-132 upregulated the expression of 
A
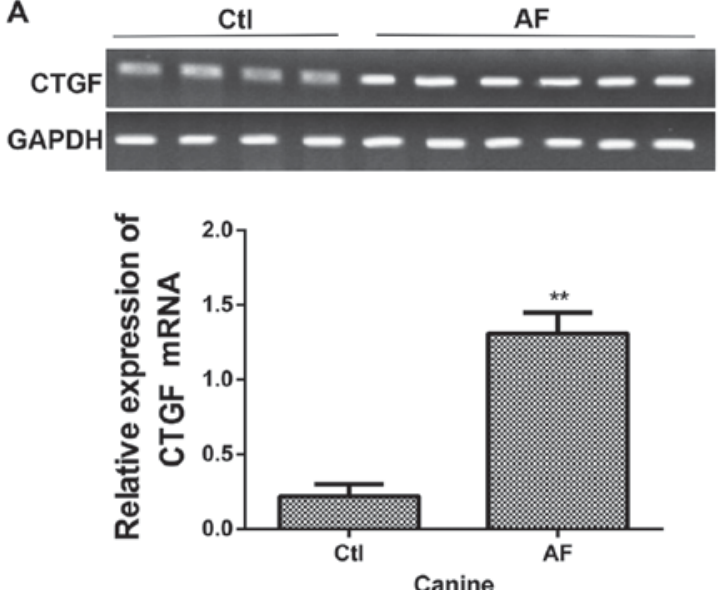

C
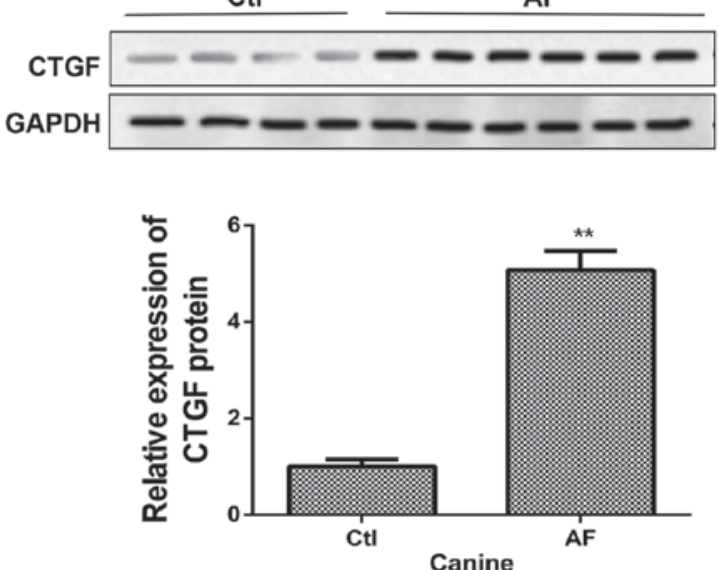

B
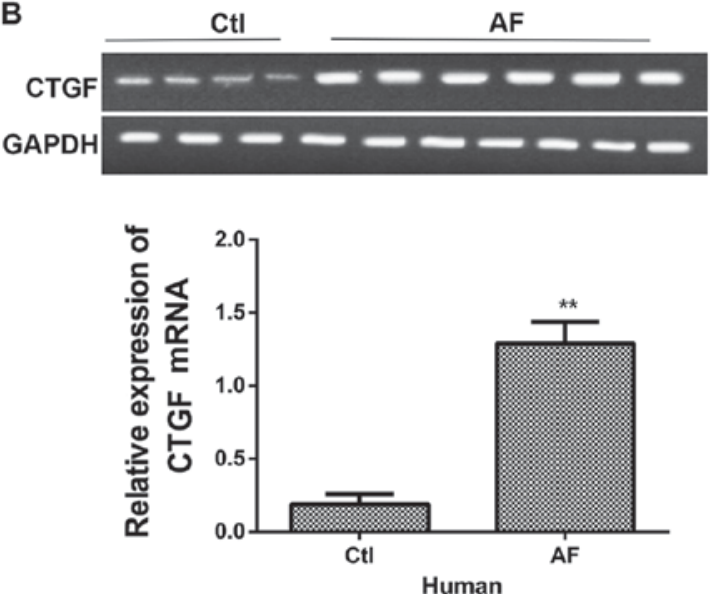

D
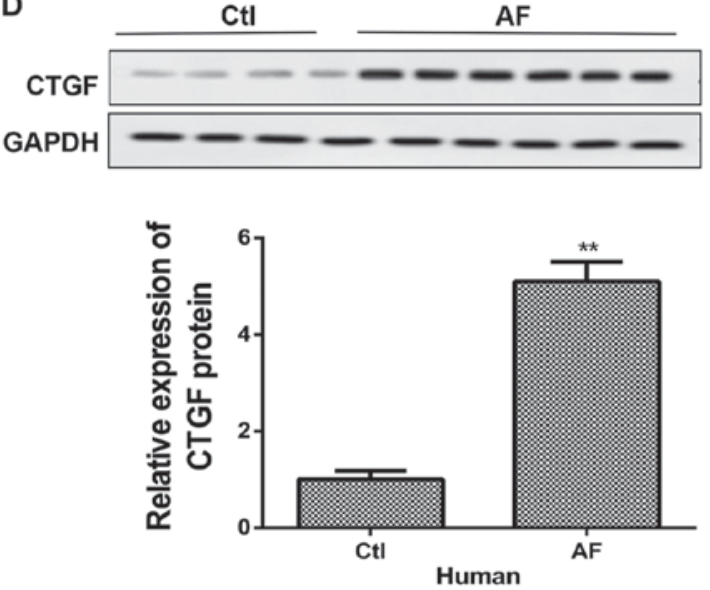

Figure 2. CTGF mRNA and protein expression levels are upregulated in canine and human samples with atrial fibrillation. (A) CTGF mRNA expression in the left atrium of dogs with or without AF and (B) CTGF mRNA expression in the right atrial appendage of patients with or without AF, detected via reverse transcription-quantitative polymerase chain reaction. (C) CTGF protein expression in the left atrium of dogs with or without AF and (D) CTGF protein expression in the right atrial appendage of patients with or without AF, detected via western blotting. Dogs with AF induced by animal pacemaker ( $\mathrm{n}=6$ ), no-paced sham dogs served as control $(n=4)$. Patients with AF $(n=6)$, patients with sinus rhythm served as control ( $n=4)$. Representative gels were from 6 different AF samples and 4 control samples. Values were normalized to GAPDH. Data are represented as the mean \pm standard deviation of the pooled samples. ${ }^{* *} \mathrm{P}<0.01$ vs. control group. AF, atrial fibrillation; CTGF, connective tissue growth factor; Ctl, control.

miR-132 and downregulated the expression of CTGF mRNA in the cardiac fibroblasts compared with $\mathrm{NC}$ group. In addition, the expression of miR-132 decreased significantly and the expression of CTGF mRNA increased in cardiac fibroblasts transfected with miR-132 inhibitor, compared with NC transfected group (Fig. 4A). This was verified by statistical analysis, presented in Fig. 4B. Furthermore, the expression of CTGF protein levels was downregulated when cardiac fibroblasts were transfected with miR-132 mimics, compared with NC. However, the cardiac fibroblasts transfected with miR-132 inhibitor exhibited the opposite trend (Fig. 4C). Subsequently, the statistical analysis of the expression of CTGF at protein level demonstrated similar results (Fig. 4D).

$C T G F$ is a direct target of miR-132. To further confirm that CTGF was a direct target of miR-132, a dual-luciferase reporter system was used. Co-expression of miR-132 significantly inhibited the luciferase reporter activity of the wild-type 3'-UTR, however not the mutant 3'-UTR in cardiac fibroblasts (Fig. 4E). The results indicated that miR-132 suppressed CTGF expression by binding to its 3'-UTR.

\section{Discussion}

The primary findings of the present study were as follows. Firstly, the expression of miR-132 was observed to be decreased, whereas the expression of CTGF increased significantly in dogs or patients with AF. Upregulation of miR-132 and CTGF was observed in Ang II-treated cardiac fibroblasts, demonstrating their key role in fibrosis. The expression of CTGF was downregulated when cardiac fibroblasts were transfected with miR-132 mimics, whereas cardiac fibroblasts transfected with miR-132 inhibitor demonstrated the opposite tendency, suggesting miR-132 may downregulate the expression of CTGF.

The miRNA, miR-132, has been identified as a target of the transcription factor, CREB, through a genome-wide screen (32). Analysis of the miRNA expression profile in cardiac fibroblasts treated with Ang II, suggests that miR-132 is markedly upregulated (17). A previous study additionally revealed that miR-132 is upregulated by Ang II in HEK293N cells, and is associated with various cardiovascular diseases including myocardial infarction, hypertrophy and fibrosis (33). Additionally, miR-132 has been demonstrated as an angiogenic 

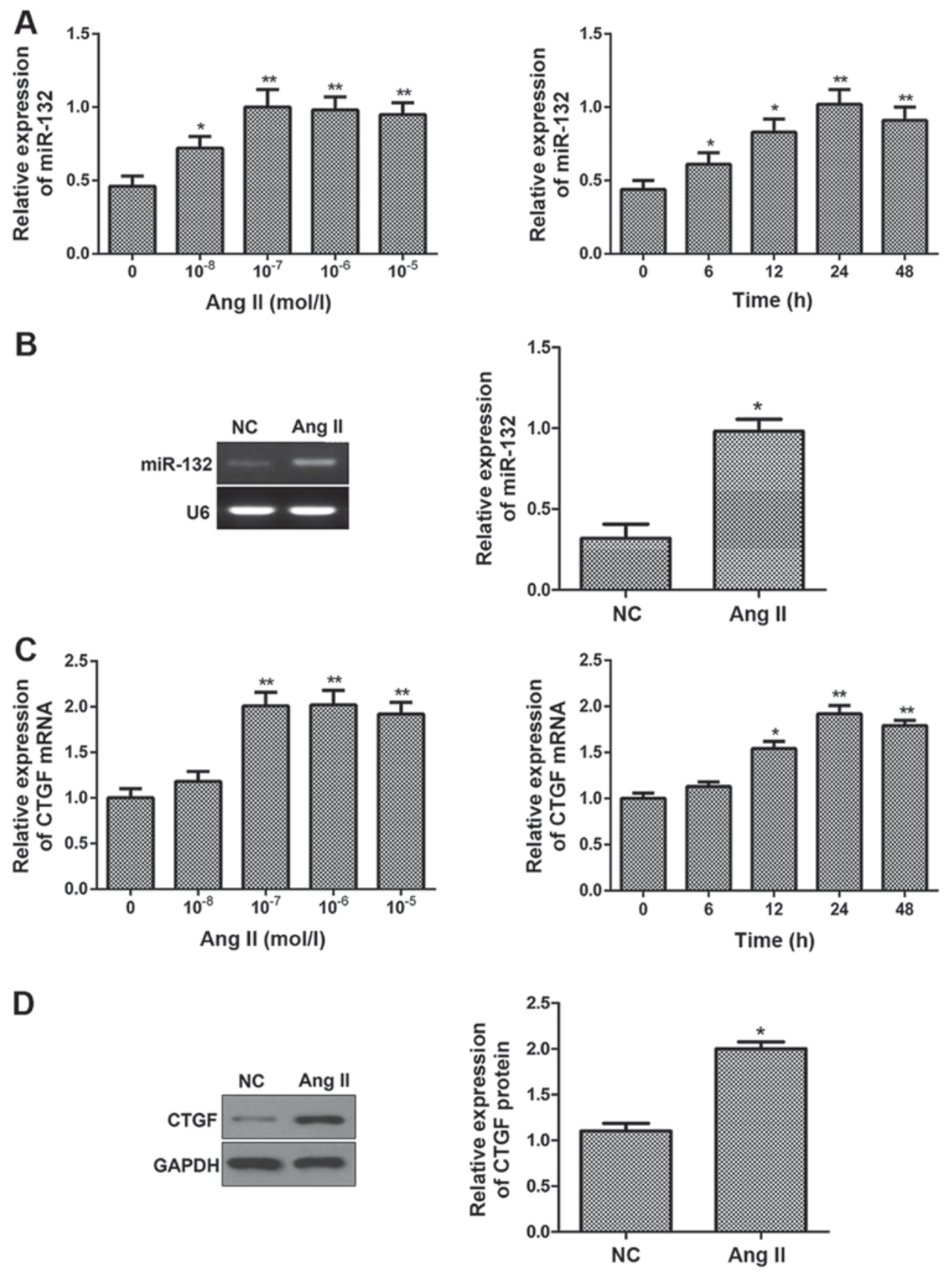

Figure 3. miR-132 and CTGF expression levels are upregulated in cardiac fibroblasts of adult rats treated with Ang II (10 $\left.{ }^{-7} \mathrm{~mol} / 1,24 \mathrm{~h}\right)$. (A) Expression of miR-132 mRNA in cardiac fibroblasts incubated with increasing concentrations of Ang II $\left(10^{-8}, 10^{-7}, 10^{-6}, 10^{-5} \mathrm{~mol} / \mathrm{l}\right)$ for $24 \mathrm{~h}$ or treated for different time lengths $(6,12,24,48 \mathrm{~h})$ with $10^{-7} \mathrm{~mol} / 1$ Ang II. ${ }^{*} \mathrm{P}<0.05$ and ${ }^{* *} \mathrm{P}<0.01$ vs. 0 mol/1 group. (B) Expression levels of miR-132 in the cardiac fibroblasts with or without Ang II detected by reverse transcription-quantitative polymerase chain reaction. "P<0.05 vs. NC group. (C) Expression of CTFG mRNA in cardiac fibroblasts incubated with increasing concentrations of Ang $\operatorname{II}\left(10^{-8}, 10^{-7}, 10^{-6}, 10^{-5} \mathrm{~mol} / \mathrm{l}\right)$ for $24 \mathrm{~h}$ or treated for different time lengths $(6,12,24,48 \mathrm{~h})$ with $10^{-7}$ mol/1 Ang II. " $\mathrm{P}<0.05$ and ${ }^{* *} \mathrm{P}<0.01$ vs. 0 mol/1 group. (D) CTGF protein expression measured by western blotting in the cardiac fibroblasts with or without Ang II. " $\mathrm{P}<0.05$ vs. Ang II-non-treated group. All values are expressed as the mean \pm standard deviation $(\mathrm{n}=4)$. Ang II, angiotensin II; NC, Ang II-non-treated negative control group; miR-132, microRNA-132; CTFG, connective tissue growth factor.

switch and a growth promoter in the endothelium by activating Ras signaling (34). In the present study, the increase of miR-132 levels in samples treated with Ang II was consistent with previous studies. Furthermore, Ang II, which is mediated via Ang II receptor type 1, is used to induce cardiac fibrosis (35). These findings indicated that miR-132 exhibits a key role in cardiac fibrosis. However, the genes which are regulated by miR-132 in cardiac fibrosis are still unknown.

The present study demonstrated that CTGF may be regulated by miR-132 and may therefore exhibit an anti-fibrotic effect in cardiac fibroblasts. It was demonstrated that in Ang II-induced fibrosis, Ang II increased CTGF gene expression and CTGF may be a mediator of the mechanisms induced by Ang II (36). Adam et al (37) demonstrated that CTGF increases in the left atrium of patients with AF and is associated with increased fibrosis and increased levels of Ang II (37). Li et al (24) revealed that the expression of CTGF is elevated in atrial tissue of patients with AF and the upregulation of CTGF contributes to atrial structural remodeling, thereby leading to AF (24). The present study suggested that the expression of 

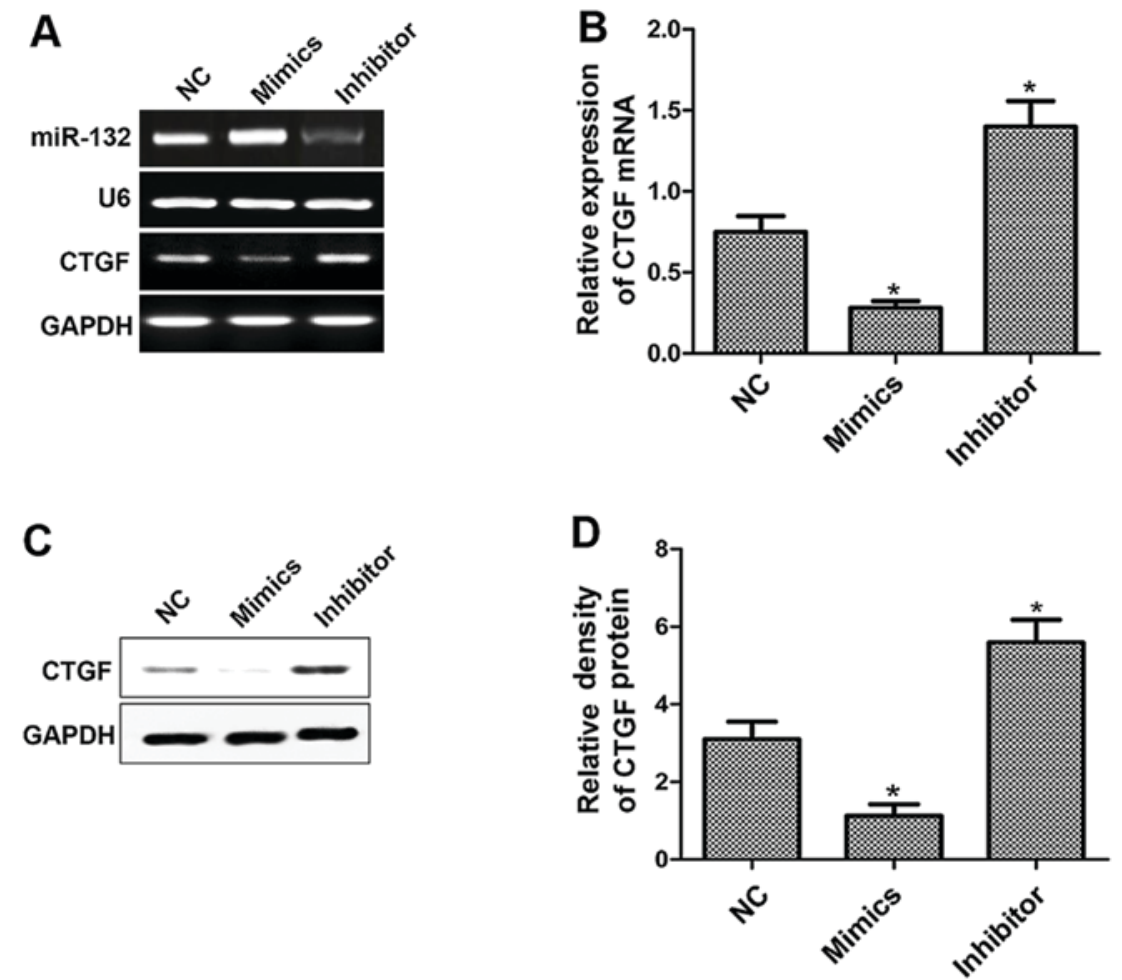

$\mathbf{E}$
CTGF 3'UTR WT 5'...UUUCUACUUUGAUAUGACUGUUU...
miR-132 3' GCUGGUACCGACAUCUGACAAU
CTGF 3'UTR Mut 5'...UUUCUACUUUGAUAUCUGACAAU...

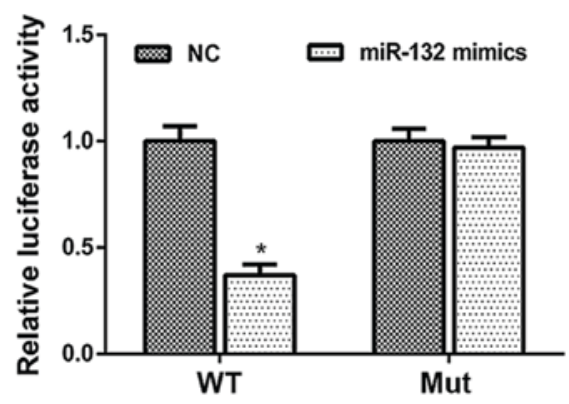

Figure 4. miR-132 directly targets CTGF in cardiac fibroblasts of adult rats. (A) Expression levels of miR-132 and CTGF mRNA in the cardiac fibroblasts transfected with miR-132 mimics $(50 \mathrm{nM})$, inhibitor $(50 \mathrm{nM})$ or NC (50 nM) for $48 \mathrm{~h}$. (B) A graphical representation of CTGF mRNA expression profiles. (C) CTGF protein expression detected by western blotting in the cardiac fibroblasts transfected with miR-132 mimics, inhibitor or NC. (D) A graphical representation of CTGF protein expression profiles. (E) Prediction of miR-132 binding sites in the 3'-UTR of CTGF gene. Potential binding site is highlighted in red. Luciferase reporter assays in cardiac fibroblasts. " $\mathrm{P}<0.05$ vs. $\mathrm{NC}$ group. All values are expressed as the mean \pm standard deviation (n=4). miR-132, microRNA-132; CTFG, connective tissue growth factor; NC, negative control scrambled group; 3'-UTR, 3'-untranslated region; WT, wild-type; Mut, mutated group.

CTGF was upregulated in the dogs or patients with AF and the CTGF gene was additionally expressed at a high level in Ang II treated cardiac fibroblasts. The results were in accordance with the aforementioned studies.

It has previously been demonstrated that miR-133a acts to regulate CTGF expression as a repressor in the regulation of cardiac fibrosis (38). miR-133 or miR-30c decrease CTGF expression levels to regulate structural alterations in the extracellular matrix (ECM) of the myocardium (27). The direct binding of miR-133 to CTGF 3'UTR has already been demonstrated by the use of luciferase assays (27). In addition, miR-18a and miR-19b regulate CTGF expression in the heart to control the surrounding ECM of cardiomyocytes (39). The present study, to the best of our knowledge, is the first to reveal that CTGF may be regulated by miR-132 and alleviate fibrosis in the heart. The findings of the present study help to expand the knowledge and understanding of the roles and association of miR-132 and CTGF in AF.

In conclusion, the results of the present study demonstrated that miR-132 was downregulated in canine or human samples with AF. miR-132 may alleviate fibrosis in cardiac fibroblasts by downregulating the expression of CTGF. The findings suggest a novel mechanism for future research to explore the function of miR-132 and CTGF in AF-induced fibrosis and may provide a potential therapeutic target for the treatment of $\mathrm{AF}$ in the future. 


\section{References}

1. Wang TJ, Larson MG, Levy D, Vasan RS, Leip EP, Wolf PA, D'Agostino RB, Murabito JM, Kannel WB and Benjamin EJ: Temporal relations of atrial fibrillation and congestive heart failure and their joint influence on mortality: The Framingham heart study. Circulation 107: 2920-2925, 2003.

2. Allessie M, Ausma J and Schotten U: Electrical, contractile and structural remodeling during atrial fibrillation. Cardiovasc Res 54: 230-246, 2002.

3. Burstein B and Nattel S: Atrial fibrosis: Mechanisms and clinical relevance in atrial fibrillation. J Am Coll Cardiol 51: 802-809, 2008.

4. Schotten U, Verheule S, Kirchhof $P$ and Goette A: Pathophysiological mechanisms of atrial fibrillation: A translational appraisal. Physiol Rev 91: 265-325, 2011.

5. Takeda N, Manabe I, Uchino Y, Eguchi K, Matsumoto S, Nishimura S, Shindo T, Sano M, Otsu K, Snider P, et al: Cardiac fibroblasts are essential for the adaptive response of the murine heart to pressure overload. J Clin Invest 120: 254-265, 2010.

6. Porter KE and Turner NA: Cardiac fibroblasts: At the heart of myocardial remodeling. Pharmacol Ther 123: 255-278, 2009.

7. Li D, Shinagawa K, Pang L, Leung TK, Cardin S, Wang Z and Nattel S: Effects of angiotensin-converting enzyme inhibition on the development of the atrial fibrillation substrate in dogs with ventricular tachypacing-induced congestive heart failure. Circulation 104: 2608-2614, 2001.

8. Ren J, Yang M, Qi G, Zheng J, Jia L, Cheng J, Tian C, Li H, Lin X and Du J: Proinflammatory protein CARD9 is essential for infiltration of monocytic fibroblast precursors and cardiac fibrosis caused by Angiotensin II infusion. Am J Hypertens 24: 701-707, 2011

9. Lijnen PJ, van Pelt JF and Fagard RH: Stimulation of reactive oxygen species and collagen synthesis by angiotensin II in cardiac fibroblasts. Cardiovasc Ther 30: e1-e8, 2012.

10. Mehal WZ, Iredale J and Friedman SL: Scraping fibrosis: Expressway to the core of fibrosis. Nat Med 17: 552-553, 2011.

11. Bartel DP: MicroRNAs: Target recognition and regulatory functions. Cell 136: 215-233, 2009.

12. Chau BN and Brenner DA: What goes up must come down: The emerging role of microRNA in fibrosis. Hepatology 53: 4-6, 2011.

13. Adam O, Löhfelm B, Thum T, Gupta SK, Puhl SL, Schäfers HJ, Böhm M and Laufs U: Role of miR-21 in the pathogenesis of atrial fibrosis. Basic Res Cardiol 107: 278, 2012.

14. Li H, Li S, Yu B and Liu S: Expression of miR-133 and miR-30 in chronic atrial fibrillation in canines. Mol Med Rep 5: 1457-1460, 2012.

15. Van Rooij E, Sutherland LB, Thatcher JE, DiMaio JM, Naseem RH, Marshall WS, Hill JA and Olson EN: Dysregulation of microRNAs after myocardial infarction reveals a role of miR-29 in cardiac fibrosis. Proc Natl Acad Sci USA 105: 13027-13032, 2008.

16. Vo N, Klein ME, Varlamova O, Keller DM, Yamamoto T, Goodman RH and Impey S: A cAMP-response element binding protein-induced microRNA regulates neuronal morphogenesis. Proc Natl Acad Sci USA 102: 16426-16431, 2005.

17. Jiang $X$, Ning $Q$ and Wang J: Angiotensin II induced differentially expressed microRNAs in adult rat cardiac fibroblasts. J Physiol Sci 63: 31-38, 2013.

18. Jin W, Reddy MA, Chen Z, Putta S, Lanting L, Kato M, Park JT, Chandra M, Wang C, Tangirala RK and Natarajan R: Small RNA sequencing reveals microRNAs that modulate angiotensin II effects in vascular smooth muscle cells. J Biol Chem 287: $15672-15683,2012$.

19. Park JK, Henry JC, Jiang J, Esau C, Gusev Y, Lerner MR, Postier RG, Brackett DJ and Schmittgen TD: miR-132 and miR-212 are increased in pancreatic cancer and target the retinoblastoma tumor suppressor. Biochem Biophys Res Commun 406: 518-523, 2011.

20. Luo J, Meng C, Tang Y, Zhang S, Wan M, Bi Y and Zhou X: miR-132/212 cluster inhibits the growth of lung cancer xenografts in nude mice. Int J Clin Exp Med 7: 4115-4122, 2014.

21. Shi-Wen X, Leask A and Abraham D: Regulation and function of connective tissue growth factor/CCN2 in tissue repair, scarring and fibrosis. Cytokine Growth Factor Rev 19: 133-144, 2008.
22. Lendeckel U, Wolke C and Goette A: Atrial fibrillation and fibrosis: Role of connective tissue growth factor. Europace 14: 1079-1080, 2012

23. Ko WC, Hong CY, Hou SM, Lin CH, Ong ET, Lee CF, Tsai CT and Lai LP: Elevated expression of connective tissue growth factor in human atrial fibrillation and angiotensin II-treated cardiomyocytes. Circ J 75: 1592-1600, 2011.

24. Li Y, Jian Z, Yang ZY, Chen L, Wang XF, Ma RY and Xiao YB: Increased expression of connective tissue growth factor and transforming growth factor-beta-1 in atrial myocardium of patients with chronic atrial fibrillation. Cardiology 124: 233-240, 2013.

25. Chen MM, Lam A, Abraham JA, Schreiner GF and Joly AH: CTGF expression is induced by TGF- beta in cardiac fibroblasts and cardiac myocytes: A potential role in heart fibrosis. J Mol Cell Cardiol 32: 1805-1819, 2000.

26. OhnishiH,Oka T,KusachiS,Nakanishi T, TakedaK,NakahamaM, Doi M, Murakami T, Ninomiya Y, Takigawa M and Tsuji T: Increased expression of connective tissue growth factor in the infarct zone of experimentally induced myocardial infarction in rats. J Mol Cell Cardiol 30: 2411-2422, 1998.

27. Duisters RF, Tijsen AJ, Schroen B, Leenders JJ, Lentink V, van der Made I, Herias V, van Leeuwen RE, Schellings MW, Barenbrug $\mathrm{P}$, et al: miR-133 and miR-30 regulate connective tissue growth factor Implications for a role of microRNAs in myocardial matrix remodeling. Circ Res 104: 170-178, 2009.

28. Nakatani Y, Nishida K, Sakabe M, Kataoka N, Sakamoto T, Yamaguchi Y, Iwamoto J, Mizumaki K, Fujiki A and Inoue H: Tranilast prevents atrial remodeling and development of atrial fibrillation in a canine model of atrial tachycardia and left ventricular dysfunction. J Am Coll Cardiol 61: 582-588, 2013.

29. Li S, Zhu J, Zhang W, Chen Y, Zhang K, Popescu LM, Ma X, Lau WB, Rong R, Yu X, et al: Signature microRNA expression profile of essential hypertension and its novel link to human cytomegalovirus infection. Circulation 124: 175-184, 2011.

30. Livak KJ and Schmittgen TD: Analysis of relative gene expression data using real-time quantitative PCR and the 2(-Delta Delta C(T)) method. Methods 25: 402-408, 2001.

31. Guo Y, Lu X and Wang H: Downregulation of miR-18a induces CTGF and promotes proliferation and migration of sodium hyaluronate treated human corneal epithelial cells. Gene 591: 129-136, 2016.

32. Vo N, Klein ME, Varlamova O, Keller DM, Yamamoto T, Goodman RH and Impey S: A cAMP-response element binding protein-induced microRNA regulates neuronal morphogenesis. Proc Natl Acad Sci USA 102: 16426-16431, 2005.

33. Jeppesen PL, Christensen GL, Schneider M, Nossent AY, Jensen HB, Andersen DC, Eskildsen T, Gammeltoft S, Hansen JL and Sheikh SP: Angiotensin II type 1 receptor signalling regulates microRNA differentially in cardiac fibroblasts and myocytes. Br J Pharmacol 164: 394-404, 2011.

34. Anand S, Majeti BK, Acevedo LM, Murphy EA, Mukthavaram R, Scheppke L, Huang M, Shields DJ, Lindquist JN, Lapinski PE, et al: MicroRNA-132-mediated loss of p120RasGAP activates the endothelium to facilitate pathological angiogenesis. Nat Med 16: 909-914, 2010.

35. Zhang Y, Huang XR, Wei LH, Chung AC, Yu CM and Lan HY: miR-29b as a therapeutic agent for angiotensin II-induced cardiac fibrosis by targeting TGF- $\beta / \mathrm{Smad} 3$ signaling. Mol Ther 22: 974-985, 2014.

36. Rupérez M, Lorenzo Ó, Blanco-Colio LM, Esteban V, Egido J and Ruiz-Ortega M: Connective tissue growth factor is a mediator of angiotensin II-induced fibrosis. Circulation 108: 1499-1505, 2003.

37. Adam O, Frost G, Custodis F, Sussman MA, Schäfers HJ, Böhm M and Laufs U: Role of Rac1 GTPase activation in atrial fibrillation. J Am Coll Cardiol 50: 359-367, 2007.

38. Angelini A, Li Z, Mericskay M and Decaux JF: Regulation of connective tissue growth factor and cardiac fibrosis by an SRF/microRNA-133a axis. PLoS One 10: e0139858, 2015.

39. van Almen GC, Verhesen W, van Leeuwen RE, van de Vrie M, Eurlings C, Schellings MW, Swinnen M, Cleutjens JP, van Zandvoort MA, Heymans S and Schroen B: MicroRNA-18 and microRNA-19 regulate CTGF and TSP-1 expression in age-related heart failure. Aging Cell 10: 769-779, 2011. 